\title{
Systematic review and meta-analysis of the effect of transcutaneous electrical acupoint stimulation on gastrointestinal function after laparoscopic surgery
}

\author{
Sheng Zhang ${ }^{1}$, Wenping Guo ${ }^{2}$, Yalou Jiao ${ }^{3}$, Xiangyan Guo ${ }^{1}$, Lijia Xu ${ }^{1}$, Hui Gao ${ }^{1}$ \\ ${ }^{1}$ Department of Emergency, Xingtai People's Hospital of Hebei Province, Xingtai, China; ${ }^{2}$ Rehabilitation Department of Traditional Chinese \\ Medicine, Xingtai People's Hospital of Hebei Province, Xingtai, China; ${ }^{3}$ Department of Reproductive Medicine, Xingtai People's Hospital of Hebei \\ Province, Xingtai, China \\ Contributions: (I) Conception and design: All authors; (II) Administrative support: All authors; (III) Provision of study materials or patients: All \\ authors; (IV) Collection and assembly of data: All authors; (V) Data analysis and interpretation: All authors; (VI) Manuscript writing: All authors; (VII) \\ Final approval of manuscript: All authors. \\ Correspondence to: Hui Gao. Department of Emergency, Xingtai People’s Hospital of Hebei Province, Xingtai 054000, China. Email: zs6600a@163.com.
}

Background: Postoperative gastrointestinal dysfunction (PGD) leading to nausea, vomiting, and abdominal distension are common complications after laparoscopic surgery for abdominal diseases. However, drugs cannot completely stop PGD. Over the years, transcutaneous electrical acupoint stimulation (TEAS) therapy has shown potential in preventing PGD, but there is no medical evidence that TEAS represents the best choice for the treatment of PGD. This network meta-analysis sought to evaluate the effectiveness of TEAS therapy in preventing PGD in patients after abdominal laparoscopic surgery.

Methods: Articles (published from the establishment of the databases to July 2021) were retrieved from the following databases: PubMed/Medline, Cochrane Library, Web of Science, Embase, China Biomedical Literature Database (CBM), China Technical Journal VIP Database (CQVIP), China Knowledge Infrastructure (CNKI), and the Wanfang Database. The Cochrane risk of bias tool was used to evaluate the quality of the included studies, and a network meta-analysis was performed using RevMan 5.20.

Results: A total of 7 randomized controlled trials, comprising 440 TEAS-treated patients and 468 control patients, were included in the meta-analysis. The meta-analysis showed that there was no significant difference between the TEAS treatment group and the control group in relation to postoperative nausea and vomiting [relative risk $(\mathrm{RR})=0.66 ; 95 \%$ confidence interval $(\mathrm{CI}): 0.37-1.21 ; \mathrm{P}=0.18$ ], postoperative abdominal distension ( $\mathrm{RR}=0.53$; 95\% CI: $0.40-0.72 ; \mathrm{P}<0.0001$ ), the time of first postoperative fart (imply gastrointestinal motility) [mean difference $(\mathrm{MD})=-7.31 ; 95 \% \mathrm{CI}:-11.33$ to $-3.30 ; \mathrm{P}=0.0004$ ], and the time of first postoperative bowel movement $(\mathrm{MD}=-5.28 ; 95 \% \mathrm{CI}:-7.23$ to $-3.33 ; \mathrm{P}<0.0001)$; there were significant differences among these 3 indicators.

Discussion: We found that TEAS can promote postoperative fart and bowel movement, and has good clinical value in promoting postoperative gastrointestinal function recovery.

Keywords: Transcutaneous electrical acupoint stimulation (TEAS); gastrointestinal disorders; laparoscopic surgery

Submitted Sep 22, 2021. Accepted for publication Nov 16, 2021.

doi: 10.21037/apm-21-3046

View this article at: https://dx.doi.org/10.21037/apm-21-3046 


\section{Introduction}

With the development of surgical techniques, most abdominal diseases can be treated with surgery. Due to the interference of surgery, patients often experience gastrointestinal dysfunction after surgery. Indeed, postoperative gastrointestinal dysfunction (PGD) has become one of the most common complications in patients who have undergone major abdominal surgery (1). Surgeons have been striving to minimize its prevalence; however, the incidence of PGD is still high, and affects $10-30 \%$ of patients who have undergone abdominal surgery $(2,3)$. The main function of the gastrointestinal tract is to digest and absorb nutrients to maintain normal physiological functions. It also plays an extremely important role in the regulation of intestinal flora. Thus, the delayed recovery of gastrointestinal function is related to the length of hospitalization and an increased risk of postoperative complications $(4,5)$. Delayed recovery also increases the medical expenses of patients, and the financial burdens placed on hospital resources.

To avoid the adverse events caused by PGD, various techniques have been studied to promote the recovery of gastrointestinal function, thereby preventing and reducing PGD. For example, early exercise (6) and reducing the use of opioids (7) can promote the recovery of gastrointestinal function, and non-steroidal anti-inflammatory drugs (NSAIDs) have also been shown to have a positive effect on the recovery of gastrointestinal function (8). However, patients are often too weak to move soon after surgery, and the side effects of NSAIDs can limit their use. Thus, there is an urgent need to explore an effective and feasible method to manage PGD. Acupuncture is a promising option that may be able to meet these needs.

Acupuncture and moxibustion is an important technology and component of traditional Chinese medicine. Acupuncture is widely used to treat a variety of diseases and physical disorders clinically (9). With the development of technology, acupuncture technology is also moving forward. Traditional acupuncture has been improved by transcutaneous electrical acupoint stimulation (TEAS). Electrodes can send electrical impulses to acupoints through the surface of the skin to achieve the purpose of treatment. This method has begun to be more and more widely accepted $(10,11)$. A recent cross-sectional study showed that acupuncture treatment of gastrointestinal diseases has become a common program in a number of countries (12). However, the efficacy of providing TEAS during surgery and as part of postoperative care for the recovery of gastrointestinal function remains unclear.

The application of laparoscopy reduces trauma; however, the gastrointestinal symptoms caused by PGD after surgery, such as nausea, vomiting, abdominal distension, fart, and delayed bowel movement, severely disturb patients and seriously affect the quality of patients' postoperative recovery. Thus, this study sought to investigate the effects of providing TEAS during the operation and as part of postoperative care on the recovery of gastrointestinal function in patients undergoing gastrointestinal surgery in patients undergoing laparoscopic abdominal surgery. A meta-analysis was conducted to evaluate the efficacy of TEAS in restoring gastrointestinal function after abdominal surgery. We present the following article in accordance with the PRISMA reporting checklist (available at https://dx.doi. org/10.21037/apm-21-3046).

\section{Methods}

\section{Search strategy}

The following major English biomedical databases were searched for articles (published from the establishment of the databases to July 2021): PubMed, Embase, CINAHL, AHMED, Cochrane Library, and Web of Science. The following major Chinese biomedical databases were searched for articles (published from the establishment of the databases to July 2021): China Biomedical Literature Database (CBM), China Technical Journal VIP Database (CQVIP), China Knowledge Infrastructure (CNKI), and the Wanfang Database. The following search terms were used: laparoscopic surgery, laparoscopy, transcutaneous electrical acupoint stimulation, traditional Chinese medicine, acupuncture, gastrointestinal function, and gastrointestinal dysfunction.

\section{Inclusion criteria}

Only prospective randomized controlled trials (RCTs) were included in the meta-analysis. The included participants had to have been diagnosed with appendicitis, intestinal obstruction, a uterine-related disease, cholecystitis, gallbladder stones, colorectal cancer, colon cancer, or rectal cancer, and have undergone laparoscopic surgical resection or treatment. The RCT had to include at least a study group and a control group, and provide measurable outcome indicators, such as nausea, vomiting, abdominal distension, 
first time to fart, and first time to bowel movement. Only the latest publication of any repeated studies were included in the meta-analysis.

\section{Exclusion criteria}

The following types of articles were excluded: reviews, case reports, and non-randomized controlled (nonRCTs). Articles were also excluded if the description and result indicators of TEAS were not provided. Studies that examined acupoint injection, suture embedding, transcutaneous electrical nerve stimulation, acupuncture combined with oral Chinese medicine, or other nonacupuncture-related therapies were excluded. Studies with unclear details of the acupuncture or related therapies were excluded. Studies were also excluded if the research object included other definite reasons for the cause of stomach intestinal disorders with intestinal dysfunction.

\section{Article screening and data extraction}

The searched articles were screened by two reviewers, and evaluated according to the inclusion and exclusion criteria. The full text of any articles included in the meta-analysis was obtained. The data were extracted and entered into a pre-designed spreadsheet. The extracted data included citation details, year, country, and journal title. If any disagreements arose between the reviewers, a 3 rd reviewer was consulted. If there were discrepancies in the published data, we had planned to contact the author, but this was ultimately unnecessary.

\section{Quality assessment}

The two reviewers independently use the Cochrane tool to assess the risk of bias of the articles, and a 3 rd reviewer was consulted to solve any problems. The following issues were considered: whether the random allocation method had been used correctly, whether allocation hiding had been used, whether blinding had been used for the research objects, the implementation of treatment plans, indicator observation records, and statistics, whether the data for the research results were complete, whether any deviations were caused by selective reporting, and whether there were any other biases.

\section{Statistical analysis}

The RevMan 5.20 software provided by Cochrane
Collaboration was used for the meta-analysis, and the relative risk (RD) or mean difference (MD) value and $95 \%$ confidence interval (CI) were calculated. A value $\mathrm{P}<0.05$ indicated a statistically significant difference. The heterogeneity was quantified as $\mathrm{I}^{2}$. When $\mathrm{P}>0.10$ and $\mathrm{I}^{2}<50 \%$, no heterogeneity was thought to exist between the studies, and the fixed-effects model was used; if any of the $\mathrm{I}^{2}$ and $\mathrm{P}$ value did not meet the conditions, the randomeffects model is used. When the studies were comparable and used the same outcome measures, a meta-analysis was performed. When 10 or more studies were available, a funnel-chart asymmetry test was used to assess publication bias. A sensitivity analysis was conducted to explore the source of heterogeneity.

\section{Results}

\section{Search results and study characteristics}

After retrieval, 1,013 records were screened, including 452 in English and 561 in Chinese. Of these, 816 records including reviews, case reports, and non-RCTs were excluded. After excluding reviews, case reports, and nonRCTs, 197 articles were screened. Finally, 114 articles retrieved. After reading the full text, excluding articles with low-quality and incomplete data according to the inclusion and exclusion criteria, 7 articles were ultimately identified, including 1 Chinese article and 6 English articles (see Figure 1 for more details).

In the 7 articles, 531 patients experienced nausea and vomiting, 674 experienced abdominal distension, 568 patients had data on the time of their first fart, and 471 patients had data on the time of their first bowel movement. All the selected articles had clear criteria for diagnosis, inclusion, and exclusion. The basic characteristics of the articles are shown in Table 1. Six articles were generated by random sequence, 6 articles described allocation hiding, 2 articles used the double-blind method, and 2 trials described the risk of bias in the blind-method results evaluation. All the articles described the results data completely. There was no selective reporting bias. Seven articles had a low risk of other biases. The evaluation results are shown in Figure 2.

\section{Meta-analysis results}

\section{Incidence of nausea and vomiting}

Four articles (comprising 251 TEAS and 280 control patients) studied the effects of TEAS on nausea and 


\section{Identification of studies via databases and registers}

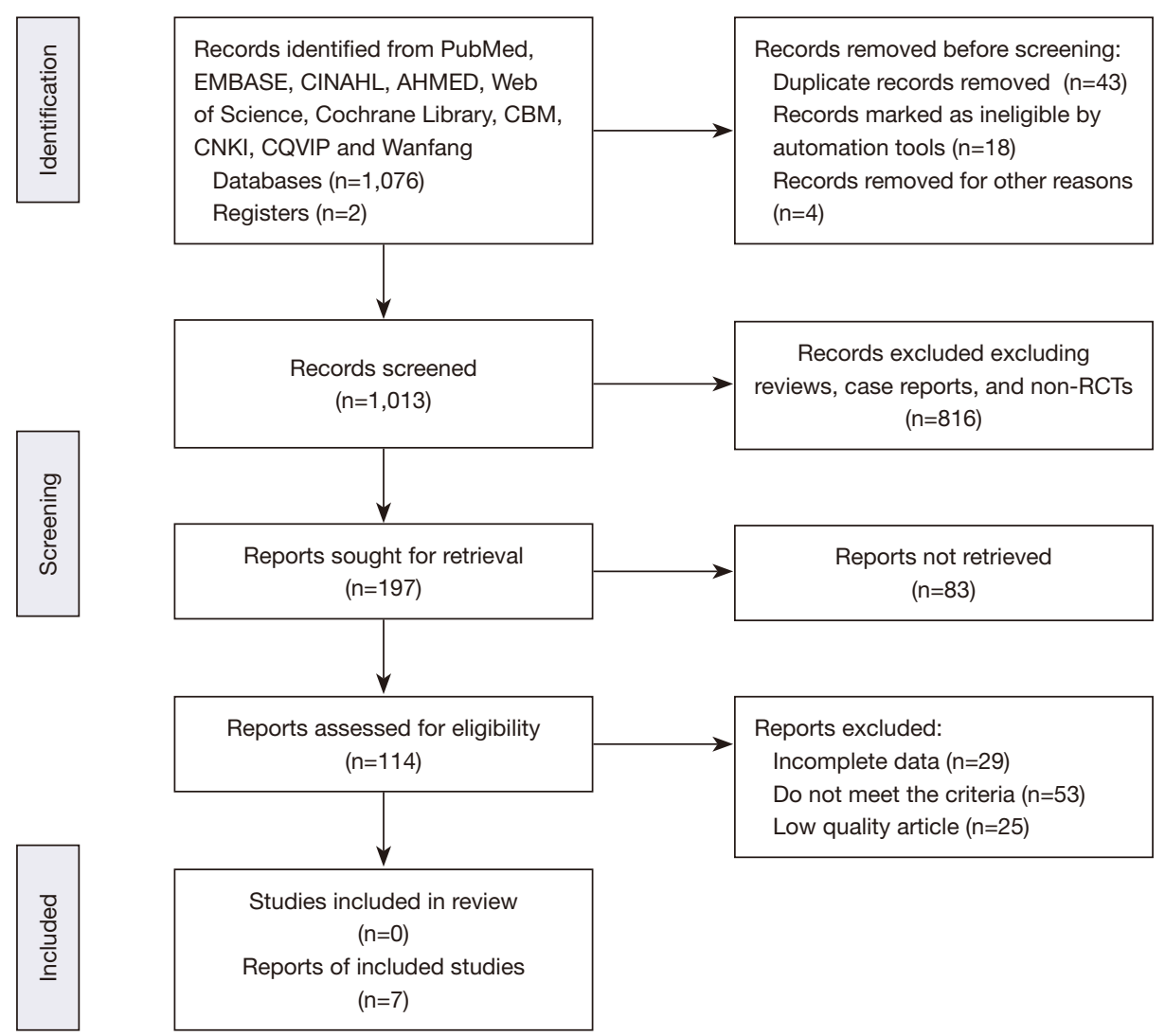

Figure 1 Flow diagram of the search, screening, and inclusion process.

Table 1 Basic characteristics of the study articles

\begin{tabular}{llllll}
\hline Author & Country & Year & Journal & TEAS (n) & Control (n) \\
\hline Gu et al. (13) & China & 2019 & European Journal of Integrative Medicine & 58 & 59 \\
Hou et al. (6) & China & 2016 & Journal of Traditional Chinese Medicine & 20 & 20 \\
Zhou et al. (14) & China & 2018 & Evidence-Based Complementary and Alternative Medicine & 43 & 29 \\
Huang et al. (10) & China & 2019 & Journal of Traditional Chinese Medicine & 140 & 140 \\
Li et al. (15) & China & 2021 & Journal of Integrative Medicine & 30 & 30 \\
Yu et al. (16) & China & 2020 & Randomized Controlled Trial & 120 & 147 \\
Zhang et al. (17) & China & 2020 & Chinese Journal of Anesthesiology &
\end{tabular}

TEAS, transcutaneous electrical acupoint stimulation.

vomiting. The analysis results showed heterogeneity between the outcome indicators $\left(\mathrm{P}=0.05, \mathrm{I}^{2}=62 \%\right)$; thus, the random-effects model was used for the combination analysis. The combined effect size RR was 0.66 (95\% CI: 0.37 to 1.21; see Figure 3). The results of the comprehensive effect size test were $\mathrm{Z}=1.34$ and $\mathrm{P}=0.18$. Thus, the meta- 


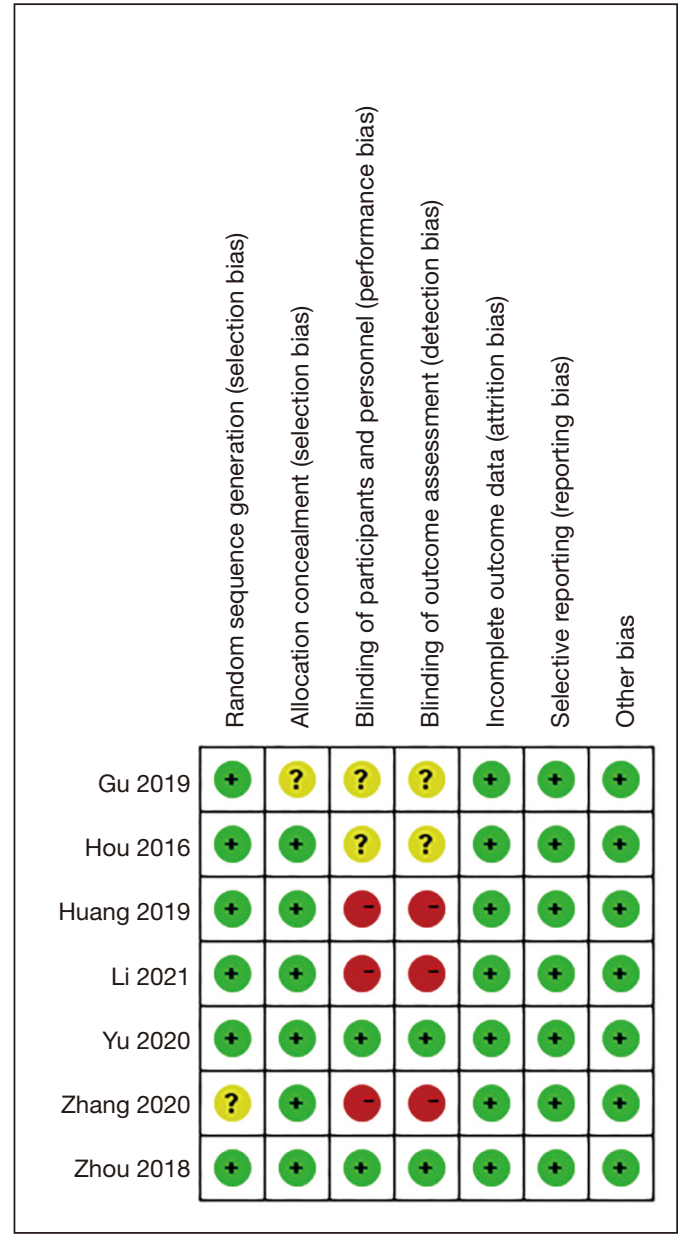

Figure 2 Literature quality evaluation details.

analysis results showed that the difference in nausea and vomiting between the TEAS treatment group and the control group was not statistically significant.

\section{Abdominal distension rate}

Four articles (comprising 323 TEAS and 351 control patients) studied the effect of TEAS on postoperative abdominal distension. The analysis results showed that there was homogeneity among the outcome indicators $\left(\mathrm{P}=0.30 ; \mathrm{I}^{2}=17 \%\right)$; thus the fixed-effects model was used for the combined analysis. The combined effect size RR was 0.53 (95\% CI: 0.40 to 0.72; see Figure 4). The results of the comprehensive effect size test were $\mathrm{Z}=4.09$ and $\mathrm{P}<0.0001$. Thus, the meta-analysis results showed that the difference in postoperative abdominal distension between the TEAS treatment group and the control group was statistically significant.

\section{Time to first postoperative fart}

Five articles (comprising 270 TEAS and 298 control patients) examined the effect of TEAS on the time of first fart after surgery. The results showed that there was heterogeneity between the outcome indicators $(\mathrm{P}=0.001$; $\mathrm{I}^{2}=77 \%$ ); thus, the random-effects model was used for the combination analysis. The combined effect size MD was -7.31 (95\% CI: -11.33 to -3.30 ; see Figure 5). The results of the comprehensive effect size test were $Z=3.57$ and $\mathrm{P}=0.0004$. Thus, the meta-analysis results showed that the difference between the TEAS treatment group and the control group in the time to first fart was statistically significant.

\section{Time to first bowel movement}

Three articles (comprising 221 TEAS and 250 control patients) examined the effect of TEAS on the time to first bowel movement after surgery. The analysis results showed homogeneity among the outcome indicators $(\mathrm{P}=0.37$; $\mathrm{I}^{2}=0 \%$ ); thus, the fixed-effects model was used for the combined analysis. The combined effect size MD was -5.28 (95\% CI: -7.23 to -3.33 ; see Figure 6). The results of the comprehensive effect size test were $\mathrm{Z}=5.31$ and $\mathrm{P}<0.0001$. Thus, the meta-analysis results showed that the difference between the TEAS treatment group and the control group in the time to the first bowel movement after surgery was statistically significant.

\section{Publication bias}

Because of the analysis of the efficacy of TEAS on the recovery of gastrointestinal function after laparoscopic surgery, the number of articles included in this study, such as nausea, vomiting, abdominal distension, fart time and bowel movement time, were all lower than the publication bias requirements $(<10)$; thus, no publication bias analysis was conducted.

\section{Risk of bias}

Six articles stated that the random sequence generation was low risk (6,10,13-16). One article had an unclear risk of bias (17). Six articles had a low risk of allocation hidden bias $(6,10,14-17)$. In relation to the risk of bias for blinding participants and researchers, 2 studies had low risk bias $(14,16)$ and 3 had high risk bias $(10,15,17)$. Five articles described the risk of bias in blinded results assessment [low risk $=2(14,16)$, and high risk $=3(10,15,17)]$. All articles had 


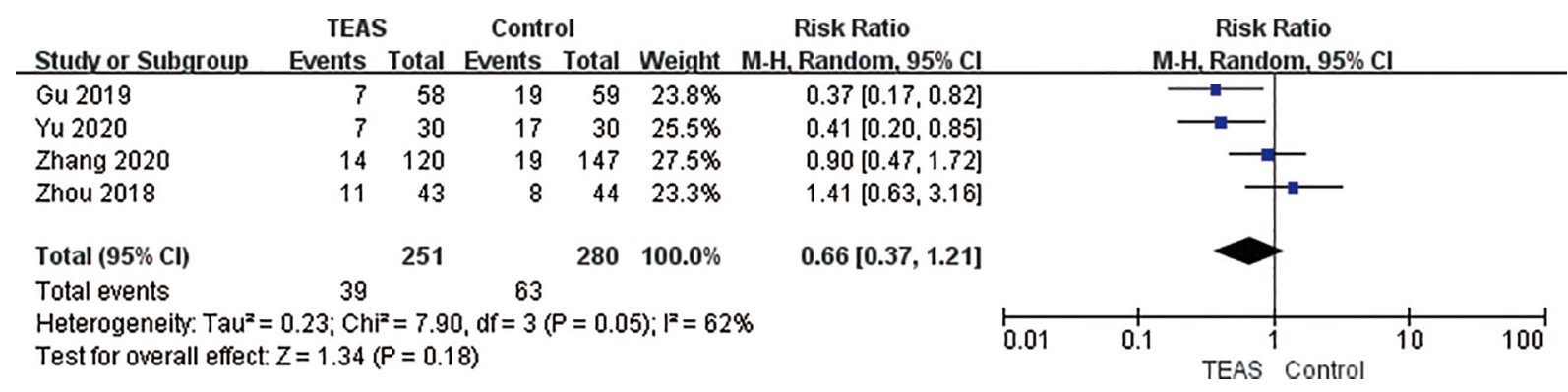

Figure 3 Forest plot of the incidence of nausea and vomiting using a fixed-effects model. Comparison of nausea and vomiting between the TEAS group and the control group after laparoscopic surgery. Statistical method: Mantel-Haenszel of the random-effects model (RR and 95\% CI). TEAS, transcutaneous electrical acupoint stimulation; RR, relative risk; CI, confidence interval.

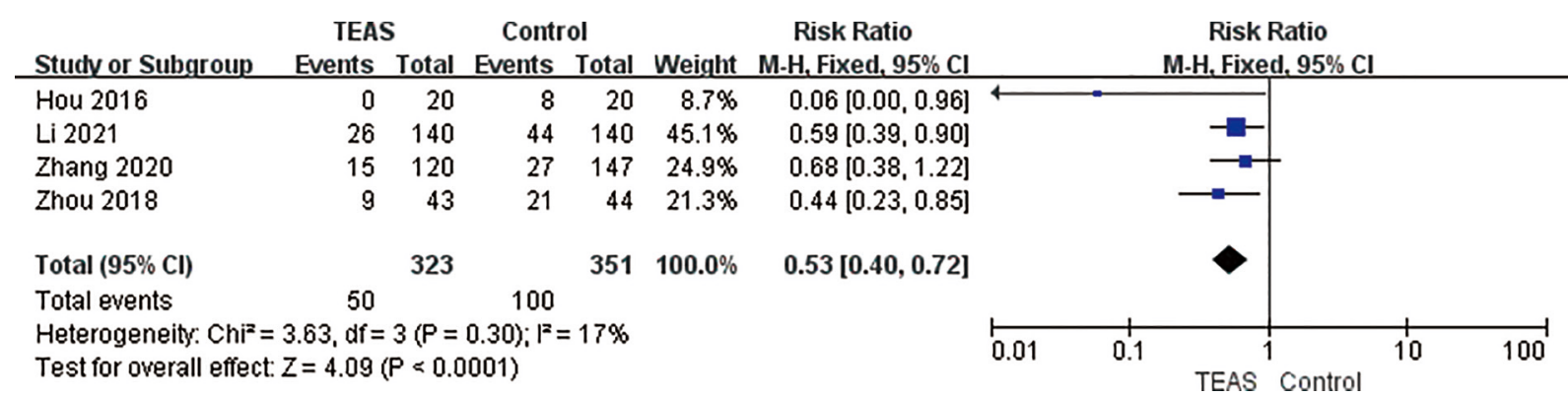

Figure 4 Forest plot of abdominal distension rate. Comparison of abdominal distension between the percutaneous TEAS group and the control group after laparoscopic surgery. Statistical method: Mantel-Haenszel of the fixed-effects model (RR and 95\% CI). TEAS, transcutaneous electrical acupoint stimulation; RR, relative risk; CI, confidence interval.

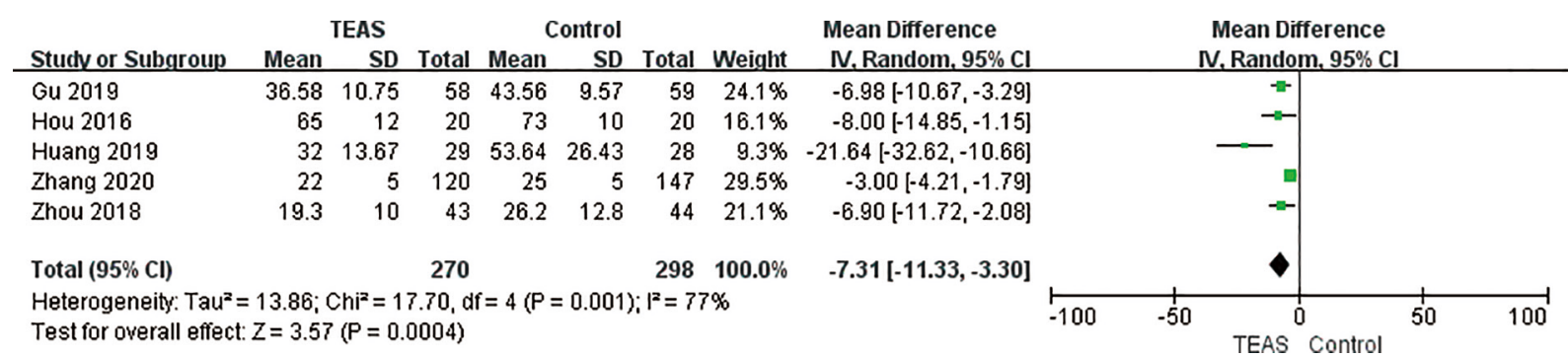

Figure 5 Forest plot of the first time to fart after surgery. Comparison of the first time to fart between the TEAS group and the control group after laparoscopic surgery. Statistical method: inverse variance of the random-effects model (MD and 95\% CI). TEAS, transcutaneous electrical acupoint stimulation; MD, mean difference; CI, confidence interval.

a low risk of bias for incomplete outcome data, selective reporting domains, and other (see Figure 7).

\section{Discussion}

Laparoscopic surgery is a minimally invasive technique that is commonly used in clinical departments, as it has some advantages, including minimal trauma, less bleeding, clear surgical field exposure, fewer postoperative complications, and the faster recovery of patients. It is widely used in a number of operations. However, in laparoscopic surgery, the effects of anesthesia and drugs, postoperative nausea and vomiting, abdominal distension, and a prolonged fart time are more common in elderly patients $(7-9,17)$. In severe 


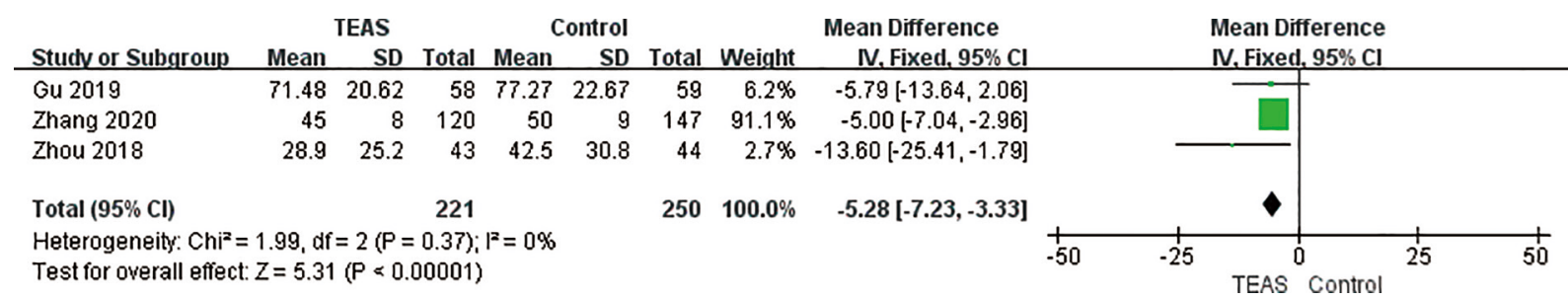

Figure 6 Forest plot of the time for first bowel movement after surgery. Comparison of the first bowel movement time between the TEAS group and the control group after laparoscopic surgery. Statistical method: inverse variance of the fixed-effects model (MD and $95 \% \mathrm{CI}$ ). TEAS, transcutaneous electrical acupoint stimulation; MD, mean difference; CI, confidence interval.

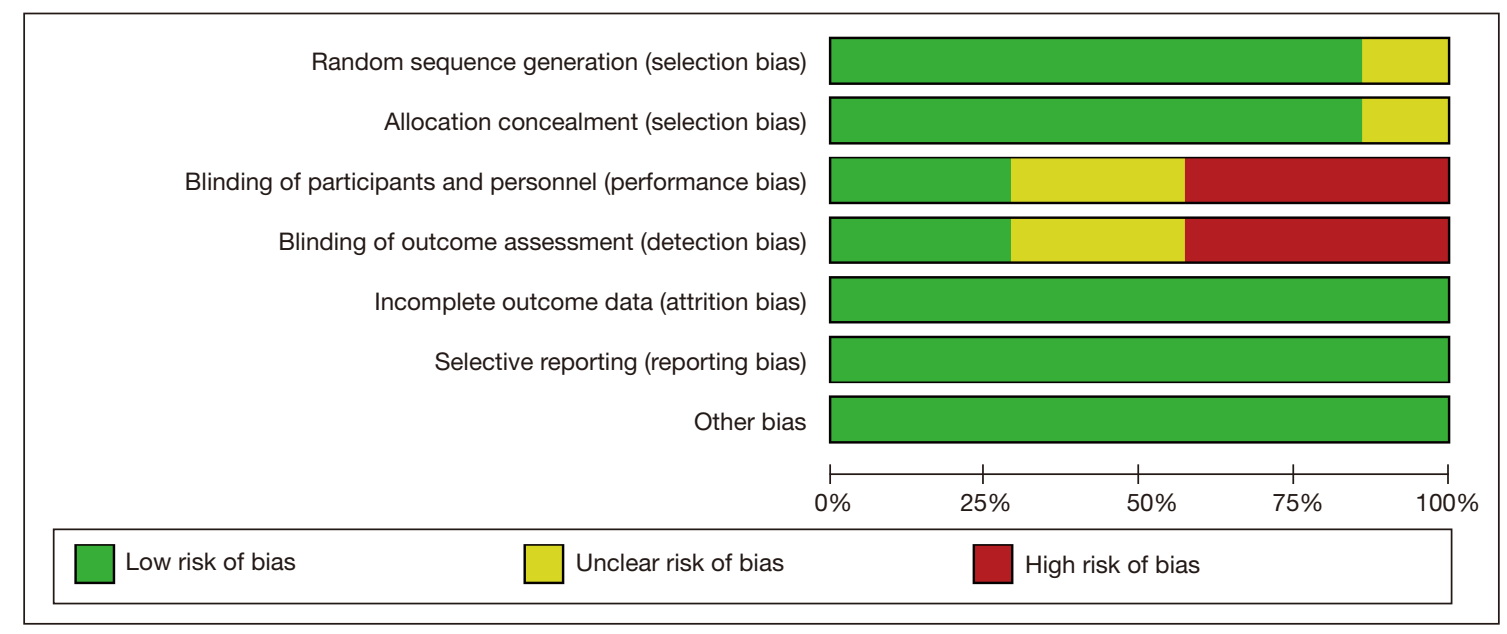

Figure 7 The intensity and distribution of the quality risk of the articles included in the study.

cases, PGD can cause water, electrolyte, acid-base balance disorders, intestinal obstruction, systemic inflammatory response syndrome and other complications, and increase the probability of secondary operations $(18,19)$. Thus, laparoscopic surgery is not conducive to the postoperative recovery of patients, prolongs hospitalization time, and increases medical expenses.

Thus, the question of how to improve and promote the recovery of gastrointestinal function after surgery has become a key issue of clinical concern. The current clinical treatment methods are mainly based on early getting out of bed exercises and drugs that promote gastrointestinal motility $(2,6,8)$. The methods are relatively simple; however, the curative effect is relatively limited, and the side effects of the drugs are many. In recent years, the effects of TEAS on the postoperative recovery of patients have gradually become a popular area of research. TEAS has a number advantages, including its convenient operation, minimal side effects, significant curative effects, and easy acceptance by patients $(1,4,12,15)$. Studies have shown that TEAS has a protective effect on the gastrointestinal tract, and that its perioperative application can promote the recovery of gastrointestinal function in patients, and reduce the occurrence of gastrointestinal complications $(18,20)$.

PGD is a common postoperative complication of abdominal surgery. As there is currently no single accepted standardized definition of PGD, it is difficult to estimate its incidence $(19,21)$. Bowel sounds include the rumbling, roaring, or grunting sounds produced by the contents of the gastrointestinal tract passing through the small intestine through a series of muscle contractions called peristalsis $(21,22)$. The recovery of bowel sounds traditionally indicates the recovery of gastrointestinal motility function after surgery (23). After the motility is restored, the patient will fart and bowel movement, and abdominal bloating disappear. Because farting and bowel movement are normal functions of the human body, it means that the patient's gastrointestinal function has returned to normal. After 
major abdominal surgery, the motility of the stomach and small intestine usually recovers within 24-48 hours (7). Thus, the absence of bowel sounds or fart 48 hours after surgery is considered a sign of PGD. The results of this study showed that pretreating patients with TEAS during the perioperative period can significantly reduce the incidence of PGD, which indicates that acupuncture intervention is a protective factor related to $\operatorname{PGD}(2,3)$. The analysis results of this study also confirmed that TEAS can promote the recovery of gastrointestinal function. These results are expected to provide a new strategy for the prevention of PGD.

However, this study had some limitations. First, as stated above, there is currently no generally accepted definition of PGD. In this study, while diagnosis of PGD had a certain theoretical basis and literature support, it still lacks clear imaging or clinical diagnosis. Thus, we only used the results of this meta-analysis as a secondary outcome indicator. Second, the effects of TEAS treatment on other internal factors were not examined, and should be examined in future studies. Finally, this study had a small sample size, short observation time, and no follow-up long-term efficacy. Thus, further research needs to be conducted addressing these issues in the future.

\section{Conclusions}

This study showed that TEAS treatment can promote the recovery of gastrointestinal function after surgery, reduce the incidence of PGD, and alleviate the suffering of patients. Given that PGD is still the main clinical and economic consequence of gastrointestinal surgery, and conventional treatments cannot effectively meet the needs of patients, TEAS may provide a feasible strategy for preventing the occurrence of PGD.

\section{Acknowledgments}

Funding: This work was supported by the Scientific Research Project of Hebei Provincial Administration of Traditional Chinese Medicine (No. 2020552).

\section{Footnote}

Reporting Checklist: The authors have completed the PRISMA reporting checklist. Available at https://dx.doi. org/10.21037/apm-21-3046
Conflicts of Interest: All authors have completed the ICMJE uniform disclosure form (available at https://dx.doi. org/10.21037/apm-21-3046). The authors have no conflicts of interest to declare.

Ethical Statement: The authors are accountable for all aspects of the work in ensuring that questions related to the accuracy or integrity of any part of the work are appropriately investigated and resolved.

Open Access Statement: This is an Open Access article distributed in accordance with the Creative Commons Attribution-NonCommercial-NoDerivs 4.0 International License (CC BY-NC-ND 4.0), which permits the noncommercial replication and distribution of the article with the strict proviso that no changes or edits are made and the original work is properly cited (including links to both the formal publication through the relevant DOI and the license). See: https://creativecommons.org/licenses/by-nc-nd/4.0/.

\section{References}

1. Liu L, Yuan X, Yang L, et al. Effect of acupuncture on hormone level in patients with gastrointestinal dysfunction after general anesthesia: A study protocol for a randomized controlled trial. Medicine (Baltimore) 2020;99:e19610.

2. Chen Y, Dong C, Lian G, et al. Dexamethasone on postoperative gastrointestinal motility: A placebocontrolled, double-blinded, randomized controlled trial. J Gastroenterol Hepatol 2020;35:1549-54.

3. Yuan HC, Xiang Q, Zhang N, et al. Acupuncture Combined with Early Enteral Nutrition on Patients with Postoperative Laparoscopic Common Bile Duct Exploration: A Prospective Randomized Trial. Chin J Integr Med 2020;26:769-75.

4. Liu Y, May BH, Zhang AL, et al. Acupuncture and Related Therapies for Treatment of Postoperative Ileus in Colorectal Cancer: A Systematic Review and MetaAnalysis of Randomized Controlled Trials. Evid Based Complement Alternat Med 2018;2018:3178472.

5. Murphy MM, Tevis SE, Kennedy GD. Independent risk factors for prolonged postoperative ileus development. J Surg Res 2016;201:279-85.

6. Hou L, Xu L, Shi Y, et al. Effect of electric acupoint stimulation on gastrointestinal hormones and motility among geriatric postoperative patients with gastrointestinal tumors. J Tradit Chin Med 2016;36:450-5. 
7. Venara A, Neunlist M, Slim K, et al. Postoperative ileus: Pathophysiology, incidence, and prevention. J Visc Surg 2016;153:439-46.

8. Paulasir S, Kaoutzanis C, Welch KB, et al. Nonsteroidal Anti-inflammatory Drugs: Do They Increase the Risk of Anastomotic Leaks Following Colorectal Operations? Dis Colon Rectum 2015;58:870-7.

9. Yang FM, Yao L, Wang SJ, et al. Current Tracking on Effectiveness and Mechanisms of Acupuncture Therapy: A Literature Review of High-Quality Studies. Chin J Integr Med 2020;26:310-20.

10. Huang W, Long W, Xiao J, et al. Effect of electrically stimulating acupoint, Zusanli (ST 36), on patient's recovery after laparoscopic colorectal cancer resection: a randomized controlled trial. J Tradit Chin Med 2019;39:433-9.

11. Chi YL, Zhang WL, Yang F, et al. Transcutaneous Electrical Acupoint Stimulation for Improving Postoperative Recovery, Reducing Stress and Inflammatory Responses in Elderly Patient Undergoing Knee Surgery. Am J Chin Med 2019;47:1445-58.

12. Wang H, Yang G, Wang S, et al. The Most Commonly Treated Acupuncture Indications in the United States: A Cross-Sectional Study. Am J Chin Med 2018. [Epub ahead of print]. doi: 10.1142/S0192415X18500738.

13. Gu S, Lang H, Gan J, et al. Effect of transcutaneous electrical acupoint stimulation on gastrointestinal function recovery after laparoscopic radical gastrectomy - A randomized controlled trial. European Journal of Integrative Medicine 2019;26:11-7.

14. Zhou D, Hu B, He S, et al. Transcutaneous Electrical Acupoint Stimulation Accelerates the Recovery of Gastrointestinal Function after Cesarean Section: A Randomized Controlled Trial. Evid Based Complement Alternat Med 2018;2018:7341920.

15. Li WJ, Gao C, An LX, et al. Perioperative transcutaneous electrical acupoint stimulation for improving postoperative gastrointestinal function: A randomized controlled trial. J Integr Med 2021;19:211-8.

16. Yu X, Zhang F, Chen B. The effect of TEAS on the quality of early recovery in patients undergoing gynecological laparoscopic surgery: a prospective, randomized, placebocontrolled trial. Trials 2020;21:43.

17. Zhang K, Zhang Y, Yu J. Effect of transcutaneous electrical acupoint stimulation on postoperative gastrointestinal function in patients undergoing laparoscopic nongastrointestinal surgery. Chinese Journal of Anesthesiology 2020;40:911-3.

18. Chen J, Tu Q, Miao S, et al. Transcutaneous electrical acupoint stimulation for preventing postoperative nausea and vomiting after general anesthesia: A meta-analysis of randomized controlled trials. Int J Surg 2020;73:57-64.

19. Fu C, Wu T, Shu Q, et al. Acupuncture therapy on postoperative nausea and vomiting in abdominal operation: A Bayesian network meta analysis. Medicine (Baltimore) 2020;99:e20301.

20. An LX, Chen X, Ren XJ, et al. Electro-acupuncture decreases postoperative pain and improves recovery in patients undergoing a supratentorial craniotomy. Am J Chin Med 2014;42:1099-109.

21. Shin KM, Park JE, Lee S, et al. Effect of siguan acupuncture on gastrointestinal motility: a randomized, sham-controlled, crossover trial. Evid Based Complement Alternat Med 2013;2013:918392.

22. Yim YK, Kang WC, Cho JH, et al. Crossover clinical trial to determine the effect of manual acupuncture at Siguan points (bilateral LI4 and LR3) on intestinal motility in healthy subjects. Am J Chin Med 2007;35:209-18.

23. Qu LZ, Zhao HC, Shen HX, et al. Electroacupuncture of both single- and multi-acupoints promotes recovery of gastrointestinal function in laparoscopic cholecystectomy patients. Zhen Ci Yan Jiu 2020;45:136-40.

(English Language Editor: L. Huleatt)
Cite this article as: Zhang S, Guo W, Jiao Y, Guo X, $\mathrm{Xu} \mathrm{L}, \mathrm{Gao} \mathrm{H}$. Systematic review and meta-analysis of the effect of transcutaneous electrical acupoint stimulation on gastrointestinal function after laparoscopic surgery. Ann Palliat Med 2021;10(11):11840-11848. doi: 10.21037/apm-21-3046 